\title{
Management of ankle injuries in the prehospital environment: A review of the literature
}

\author{
Lynsey Smit and Dr Malcolm J Boyle PhD
}

Affiliation:

Monash University, Department of Community Emergency Health and Paramedic Practice, Frankston, Victoria, Australia

\section{ABSTRACT}

\section{Introduction}

Ankle fractures have traditionally been seen as low priority cases as they do not pose an immediate life threat. Ankle fractures have the potential to create long-term mobility consequences for a person if managed inappropriately. The objective of this study was to determine the most effective way to manage ankle injuries in the prehospital environment.

\section{Methods}

A literature search was conducted of the medical related electronic databases, Ovid preMedline, Medline, Cinahl, Cochrane CENTRAL, and EMBASE from 1980 to the end of May 2013. A previously published prehospital search filter was used in each of the databases including additional keywords "fracture", "ankle", "ankle injuries", "bone fractures", and "sprains and strains". Articles were included if their primary aim was to examine the appropriate management of ankle fractures in the prehospital environment. The references of retrieved articles were also reviewed. Articles were excluded if they were not written in English.

\section{Results}

There were 531 articles located in the search. After reviewing the articles three were determined to meet the inclusion criteria. One article was set primarily in the emergency department but also included prehospital management. The remaining two articles were set in the prehospital setting. The findings suggest there is a defined set of questions to ask and specific assessment criteria for a patient with an ankle injury to determine its potential severity. Where there is neurovascular compromise anatomical realignment should be attempted.

\section{Conclusion}

A thorough initial assessment and good basic management by prehospital care providers is essential to assist in decreasing delays to definitive treatment for patients with suspected ankle fractures, dislocations or fracture/dislocations. Further research is needed to determine to most effective management strategies in order to achieve optimal outcomes for patients with ankle injuries.

\section{Keywords}

ankle fracture, management, paramedic, EMS, prehospital care, emergency care

\section{INTRODUCTION}

In 2000 at a Delphi consultation exercise the treatment and management of ankle fractures in the prehospital environment was identified as an area of prehospital practice that had gaps in research. It was subsequently placed among the priorities for prehospital research (1).

An ankle fracture is defined as a fracture of the distal tibia and/or fibula and is one of the most common lower limb fractures $(2,3)$. The ankle 
joint supports more weight per unit of area than any other joint in the body (4). Due to its importance as a supporting joint and one involved in normal body movement, management and treatment of an injured ankle should focus on achieving optimal outcomes for the patient.

The most common causes of ankle fractures are twisting injuries, falls and sports related injuries $(5,6)$. In a study conducted covering 70 different sporting activities ankle injuries were found to be the most common site of injury in 24 of the 70 sports (7). Isolated ankle injuries are not considered to be life threatening injuries, however, appropriate management is necessary to prevent potential long term mobility complications for the patient (8).

Due to an ever increasing demand for hospital beds, poor assessment and triage skills, ankle fractures are often triaged as low-priority cases (4). Due to being seen as a low-priority case there may be significant delays before definitive care is received. Depending on the severity of the fracture, definitive care such as surgical intervention may be required (4). Any injury to an ankle also causes traumatic injury to both the bone and the surrounding soft tissue (9).

Extremity fractures such as ankle fractures are often encountered in prehospital care (10). In the prehospital environment an accurate assessment of the extent of trauma to the surrounding soft tissue and bone is very challenging as diagnostic equipment such as X-rays and MRl's are unavailable. Given diagnostics difficulties in the prehospital setting and a limited range of management options, the objective of this study was to determine the most effective way to assess and manage ankle injuries in the prehospital environment.

\section{METHODS}

\section{Study Design}

This study was a review of the medical related literature to determine the best management options for prehospital ankle injury management.

\section{Procedures}

A literature search was conducted searching the following electronic databases:

Ovid Medline (1980 to end of May 2013);

Cinahl (1980 to end of May 2013); Cochrane

(1980 to end of May 2013); EMBASE (1980 to end of May 2013). A prehospital search filter was applied in order to maximise the sensitivity of the literature search for papers specifically relating to the prehospital setting (11). Additional keywords including "ankle", "ankle injuries", "bone fractures", "fracture", and "sprains and strains" were also used in the search criteria.

The reference lists of the relevant articles were reviewed with the purpose of identifying additional articles missed in the electronic database search. Articles of any study design were included if their primary aim was to examine the appropriate management of ankle injuries in the prehospital environment. Articles that were set in the emergency department (ED) were also included if their focus involved the prehospital management of ankle fractures. Letters, articles not written in English, or articles that did not examine the management of ankle injuries in the prehospital environment were excluded.

\section{RESULTS}

There were 531 articles identified located in the search. After reviewing the titles and abstracts of the identified articles, three articles met the inclusion criteria.

Two articles were clinical instructional reviews and one was a retrospective review. The articles by Lee and Porter (10) and Dean (8) and were clinical instructional reviews. Dean focussed on the treatment of ankle fractures by wilderness medics where definitive care was not readily accessible (8).

Lee and Porter on the other hand focused specifically on the prehospital management of lower limb fractures, where definitive care is more easily accessible than in a wilderness setting. Lee and Porter were of the opinion that a thorough initial assessment and appropriate basic management is of vital importance to decrease morbidity of ankle fractures (10). More research however is needed to validate the opinions of Lee and Porter.

Payne et al undertook a retrospective review of 23 patients attending the emergency department of a district general hospital. Payne and colleagues specifically focussed on the initial management of closed ankle fractures, dislocations or fracture/dislocations. It was concluded that injury recognition and triage skills are poorly preformed in the Emergency Department (ED) resulting in delays to receiving appropriate definitive care (9). Refer to Table 1 for a summary of the included articles. 


\begin{tabular}{lll} 
Author & \multicolumn{1}{c}{$\begin{array}{c}\text { Type of } \\
\text { Study and } \\
\text { Year }\end{array}$} & $\begin{array}{c}\text { Population } \\
\text { Size }\end{array}$ \\
\hline Dean (8) & $\begin{array}{l}\text { Clinical } \\
\text { Instructional } \\
\text { Review: 2009 }\end{array}$ & Not applicable \\
Payne et al (9) & $\begin{array}{l}\text { Retrospective } \\
\text { Review: 2004 }\end{array}$ & $\begin{array}{l}\text { 22 out of 23 } \\
\text { patients were } \\
\text { transported to } \\
\text { the ED by }\end{array}$ \\
& & Paramedics \\
& & Not applicable \\
Lee and Porter & $\begin{array}{l}\text { Clinical } \\
\text { Instructional } \\
\text { (10) }\end{array}$ & \\
\hline
\end{tabular}

Table 1: Summary of included studies

\section{DISCUSSION}

Early identification and management of ankle fractures is vital to ensure the patient receives definitive care without delay $(4,12)$. There is a general consensus that ankle fractures are underestimated in terms of severity $(4,9,10,12)$ and as a result patients often face lengthy delays once arriving at the emergency department. Definitive care is often delayed as a result of inadequate basic management (4). It has been emphasised that the initial assessment and basic management is of extreme importance if delays to receiving definitive treatment is to be reduced (4, $8,9)$. Paramedics may often provide the first line of medical treatment received by the patient. The initial assessment and basic management are two components requiring emphasis in the clinical approach of prehospital care. Refer to Figure 1 for clarification of where the components are situated inside a general clinical approach. This discussion will specifically look at what the initial assessment and basic management should consist of when providing prehospital care for suspected injuries.

The third component is the initial assessment, of which there are several key sub-components. These sub-components include a detailed history, noting symptoms present, obtaining a relevant past medical history, and a neurovascular status assessment $(9,10)$. The history consists of specific information with reference to how the injury occurred. Determining the mechanism of injury is of particular importance. Important questions to ask the patient are outlined in Table 2. These questions will assist in establishing how the injury occurred. If the Ottawa Ankle Rule (OAR) is used as an assessment tool these questions could also assist in determining the severity of the injury for triage purposes. The next sub-component in the initial assessment is noting the signs present. Signs to assess and note in particular are bruising, swelling, pain, deformity, and crepitus $(8,10,13)$. Relevant past medical history information is used specifically to establish if there has been a previous injury to the ankle. A vital sub-component of the initial assessment is the neurovascular assessment, the neurovascular assessment is used to establish if there is vascular compromise to the injured ankle (8-10).

For a neurovascular assessment the capillary refill time should be determined for both the uninjured and injured ankle (10). If the capillary refill time for the injured limb is longer than that of the uninjured limb then neurovascular compromise may be present. If blood flow to the injured area is compromised as a result of the fracture, dislocations or fracture/dislocations the ankle needs to be anatomically realigned or returned to the normal anatomical position once adequate analgesia has been provided. The realignment attempts to restore blood flow and prevent further soft tissue injury $(4,8,10)$. Realignment of a fractured, dislocated or fractured/dislocated ankle with neurovascular compromise present is an essential skill for prehospital care providers, especially if long transport times to hospital are a potential (9).

\section{Questions}

1. How did the injury occur?

2. When did the injury occur?

3. Why did the injury occur?

4. Can you weight bear?

5. Can the ankle be moved side to side, or up and down?

6. Did you hear or feel a crack?

Table 2: Important history questions $(10,13)$

Once the initial assessment is complete the basic management follows. Basic management of ankle injuries includes, but is not limited to, the following key treatments: cleaning of an open wound, manipulation, splinting, packaging, elevation and ice treatment $(4,8-10)$. If an open fracture is present the removal of gross contamination should to be washed or wiped away using saline or saline soaked pads (10). After removing the majority of the contamination the wound should be covered with a sterile Betadine dressing, if available (10).

The next management option is manipulation. In some cases manipulation of the ankle may be necessary before the ankle can be splinted and packaged $(8,10)$. Analgesia will usually be needed before manipulation or realignment is attempted (8-10). Options for analgesia provided by paramedics are intravenous morphine, ketamine or peripheral nerve blocks (10). An additional option also includes intravenous or intranasal Fentanyl. It is important to note that scene time should not be unnecessarily delayed in order to obtain intravenous access or 
administer regional blocks for time critical patients who have neurovascular compromise distal to the ankle joint(10). If however, the patient has an uncomplicated fracture, dislocation or fracture/dislocation with no neurovascular compromise the additional on scene time could be beneficial to obtain IV access and provide effective analgesia. If intravenous access is difficult to obtain inhalation analgesics should be considered as an alternative (10). Manipulation to obtain realignment is urgently required if the neurovascular assessment showed vascular compromise. Before and after manipulation is performed the neurovascular status must be assessed $(8,10)$. If the neurovascular assessment post manipulation shows compromise the ankle should be returned to the position in which the neurovascular status shows the least amount of compromise (10). If a neurovascular compromise is present pre-manipulation and does not improve once the joint has been manipulated the patient should be transported urgently with hospital notification (10).

After the fractured, dislocated or fractured/dislocated ankle has been realigned or manipulated it has to be splinted for transport to hospital. Splinting offers multiple benefits such as reducing pain and blood loss, as well as reducing pressure on the skin and surrounding neurovascular structures. In addition splinting also reduces the risk of causing more damage to the injured joint during transport (10). Immobilisation should be to the joint above and below the injured joint $(10,13)$. Splinting options available to paramedics are short leg box splints and short vacuum splints (10). Once the injured ankle is splinted paramedics should elevate the limb and if possible place ice packs on the injured ankle while transporting to the nearest appropriate facility $(4,8)$. An ice pack should be covered by a thin cloth when placed over the injured ankle on either the medial or lateral side. The injured ankle should be iced for approximately 20 minutes every 2 to 3 hours (14). The above measures will aid in decreasing swelling and blistering around the ankle with this decreasing the amount of time spent in the ED attempting to reduce swelling and blistering (4). The less time spent in the ED on basic management mentioned above the faster the patient will receive definitive care.

Ankle injuries are often triaged as low priority cases due to overcrowded emergency departments, therefore it is extremely important that the initial assessment and basic management is done well $(4,13)$. If the initial assessment and basic management is done poorly by prehospital care providers the process then has to be repeated in an already busy emergency department. This will contribute to further delaying definitive treatment (4).
The Ottawa Ankle Rule is currently used by clinicians and nurses in the emergency department very successfully to examine and rule out the majority of clinically insignificant fractures and to triage patients accordingly. Simpson and Stiell et al found that pending further research it may be feasible to implement the OAR for use by prehospital care providers as an assessment/triage tool $(15,16)$. The OAR is currently used by Extended Care Paramedics in Australia, New Zealand and the United Kingdom. If Advanced Life Support paramedics, as well as Extended Care Paramedics, are able to implement the OAR as part of their assessment, to establish the severity of the ankle injury, it may assist in obtaining an appropriate triage category in the emergency department. The initial assessment, basic management, and handover to emergency department staff contribute greatly to the time it takes for the patient to receive definitive care.

This study found that currently there is a lack of published evidence that directly examines the effectiveness of paramedic management of ankle fractures. Payne et al was the only study that retrospectively reviewed paramedic management of ankle fractures or dislocations. Payne et al however only reviewed 22 cases of paramedic management (9). As the population size is small and not representative of the general population the results cannot be generalised. None of the other articles directly reviewed which paramedic management strategies produce optimal patient outcomes.

This study is potentially limited as it did not include non-English articles. Journals that are not available electronically were not searched by hand. These limitations may have resulted in some potentially relevant articles being overlooked. The findings presented in this literature review should be interpreted with caution due to the lack of high-level evidence studies found and the small sample sizes in the studies located.

\section{CONCLUSION}

In conclusion, prehospital care providers should not underestimate the possible severity of ankle injuries. Through a thorough initial assessment, good basic management, and implementation of the Ottawa Ankle Rule, paramedics should be able to decrease delays to definitive treatment for patients with suspected ankle injuries. Further research is needed to determine the most effective management strategies in order to achieve optimal outcomes for patients with ankle injuries. 


\section{CONFLICT OF INTEREST}

The authors declare they have no conflict of interest

\section{REFERENCES}

1. Archer F, Woollard M, Snooks H, Evans A, Wells B, Peconi J, et al. What are the highest priorities for research in prehospital care? Results of a review and Delphi Consultation exercise - Executive Summary. Journal of Emergency Primary Health Care. 2008;6(4).

2. Lash N, Horne G, Fielden J, Devane P. Ankle fractures: functional and lifestyle outcome at 2 years. ANZ J Surg. 2002;72(10):714-30.

3. Lin CC, Moseley AM, Refshuage KM, Haas $M$, Herbert RD. Effectiveness of joint mobilisation after cast immobilisation for ankle fracture: a protocol for a randomised controlled trial. BMC Musculoskelet Disord. 2006;7:46.

4. Deasy C, Murphy D, McMahon GC, Kelly IP. Ankle fractures: emergency department management... Is there room for improvement? J Emerg Med. 2005;12:216-9.

5. Court-Brown CM, McBirnie J, Wilson G. Adult ankle fractures- an increasing problem? Acta Orthop Scand. 1998;69(1):43-7.

6. Jensen SL, Andresen BK, Mencke S, Nielsen PT. Epidemiology of ankle fractures: a prospective population-based study of 212 cases in Aalborg, Denmark. Acta Orthop Scand. 1998;69:48-50.

7. Fong DT, Hong Y, Chan LK, Yung PS, Chan KM. A systematic review on ankle injury and ankle sprain in sports. Sports Med. 2007;37:73-94.

8. Dean B. Field Management of Displaced Ankle Fractures: Techniques for Successful Reduction. Wilderness Environ Med. 2009;20(1):57-60.

9. Payne R, Kinmont JC, Moalypour SM. Initial management of closed fracture-dislocations of the ankle. Ann R Coll Surg Engl. 2004;86:17781.

10. Lee C, Porter KM. Prehospital management of lower limb fractures. Emerg Med J. 2005;22:660-3.

11. Smith E, Archer F, Burgess S. The development of an updated prehospital search filter for the Cochrane Library: Prehospital Search Filter Version 2.0. Journal of Emergency Primary Health Care. 2010;8(4).

12. Watson JAS, Hollingdale JP. Early management of displaced ankle fractures. Injury. 1992;23(2):87-8.

13. Larsen D. Assessment and management of foot and ankle fractures. Nurs Stand. 2002;17(6):37-46.
14. van den Bekerom MPJ, Struijs PAA, Blankevoort $L$, Welling $L$, van Dijk $C N$, Kerkhoffs GMMJ. What is the evidence for rest, ice, compression, and elevation therapy in the treatment of ankle sprains in adults? J Athlet Train. 2012;47(4):435-43.

15. Stiell IG, McKnight RD, Greenberg GH, McDowell I, Nair RC, Wells GA, et al. Implementation of the Ottawa ankle rules. JAMA. 1994;271(11):827-32.

16. Simpson P. Use of the Ottawa Ankle Rule by paramedics in the out-of-hospital setting. Journal of Emergency Primary Health Care. 2010;8(2).

\section{Primary Survey \\ DRABCDE + Haemorrhage check}

\begin{tabular}{|c|}
\hline$v$ \\
\hline $\begin{array}{l}\text { Secondary Survey } \\
\text { - Perfusion Status Assessment } \\
\text { - Respiratory Status Assessment } \\
\text { - Glasgow Coma Score } \\
\end{array}$ \\
\hline$\downarrow$ \\
\hline $\begin{array}{l}\text { Initial assessment } \\
\text { - History } \\
\text { - Symptoms present } \\
\text { - Past medical history } \\
\text { - Neurovascular status assessment } \\
\text { - Use of OAR to determine severity }\end{array}$ \\
\hline$\downarrow$ \\
\hline $\begin{array}{l}\text { Basic Management } \\
\text { - Cleaning of the wound } \\
\text { - Analgesia } \\
\text { - Manipulation if necessary } \\
\text { - Elinting of the wound for transport } \\
\text { - Ice treatment }\end{array}$ \\
\hline $\boldsymbol{\nu}$ \\
\hline Transport \\
\hline
\end{tabular}

Figure 1: Placement of initial assessment and basic management in clinical approach (adapted from Lee and Porter (10)) 